\title{
Where Nanophotonics and Microfluidics Meet
}

\author{
A. Scherer, E. Kartalov, F. Anderson ${ }^{+}$, M. Hochberg, T. Baehr-Jones, G. Wang, L.. \\ Dalton ${ }^{*}$ A. Jen
}

\author{
Caltech, Pasadena CA 91125, USA \\ University of Washington \\ ${ }^{+}$University of Southern California
}

\begin{abstract}
A new generation of photonic devices has recently emerged that relies on using geometries of sub-wavelength microstructures within a high refractive index contrast materials system. These geometries are used to confine and manipulate light within very small volumes. High optical field densities can be obtained within such structures, and these in turn can amplify optical nonlinearities. Moreover, many of these structures, as for example photonic crystals and slotted waveguides, can be engineered for the efficient localization of light within the low-index regions of high index contrast microstructures. When such structures are back-filled nonlinear polymers or liquids, devices can be tuned and novel phenomena can be observed. In particular, such devices are very interesting when constructed from silicon on insulator (SOI) material in which the optical waveguide also serves as a transparent electrical contact. Here we show examples of the design, fabrication and testing of optical microstructures in which the electro-optic $(\chi 2)$ and photorefractive $(\chi 3)$ nonlinearities are used for electro-optic tuning, frequency mixing, optical rectification, and high-speed switching of light.
\end{abstract}

The emergence of such lithographically defined optical devices also enables their integration into spectroscopic systems that can be miniaturized and integrated with electronic and fluidic systems for signal analysis and sample delivery. Now, we can integrate these devices into compact functional systems for spectroscopic analysis. In general, miniaturization results in the opportunity to reduce the sample volumes and improvement in the sensitivity and speed. Here, systems based on silicon on insulator technology, photonic crystal geometries, and the introduction of gain into spectroscopic systems will be presented. We have recently developed replication molding technologies which permit the integration of thousands of fluidic valves in spectroscopic microfluidic analysis systems. By integrating the fluidic and photonic devices, pico-Liter sample delivery can be combined with femto-Liter analytical volumes. Here we will describe this technology and review some preliminary applications of our fluorescence immuno-assay devices, particularly for the analysis of human blood serum for several common cancer markers. 